\section{AB0754 HOW DO WE TREAT PSORIATIC ARTHRITIS? EVIDENCE FROM A 15-YEAR MONOCENTRIC BDMARDS EXPERIENCE}

M. S. Chimenti ${ }^{1}$, M. Ferraioli ${ }^{1}$, A. D'antonio ${ }^{1}$, P. Conigliaro ${ }^{1}$, S. Ferrigno' ${ }^{1}$ A. Vendola ${ }^{1}$, P. Triggianese ${ }^{1}$, G. L. Fonti ${ }^{1}$, R. Perricone ${ }^{1} .{ }^{1}$ Rheumatology, Allergology and Clinical Immunology, University of Rome Tor Vergata, Rome, Italy

Background: Psoriatic arthritis (PsA) treatment paradigm has dramatically change during the last 15 years, improving patients clinical outcomes and quality of life. We have now the possibility of a wide option among bDMARDs with different targets: TNF-inhibitors (TNFi), anti-IL17A inhibitors and anti-p40IL12/23. However, the choice of the "best" treatment in PsA patients is still a challenge in our daily clinical practice, making treatment decision a tricking issue.

Objectives: Aim of the study was to retrospectively evaluate the distribution of clinical PsA subtypes and comorbidities among bDMARDs treated patients in the last 15 years in a monocentric cohort of the Rheumatology Unit, University of Rome Tor Vergata.

Methods: Patients affected by PsA diagnosed by the CASPAR criteria and treated with a bDMARDs, in the last 15 years were enrolled. Clinical assessment included the presence of: oligo and/or polyarthritis and axial involvement (yes/no), enthesitis (yes/no), dactylitis (yes/no), PsO and onychopathy (yes/no). Comorbidities, as cardiovascular, metabolic syndrome and kidney diseases, were registered. The overall timeframe was halved in: 1st period (2004-2011) and 2nd period (2012-2019). Chi-square test was used to analyze the distribution of clinical PsA subtypes and comorbidities.

Results: Data from 314 consecutive PsA patients treated with bDMARDs were obtained (Table 1). A total of $259(82,48 \%)$ patients were treated with TNFi, while $55(17,52 \%)$ with non-TNFi (i.e. Secukinumab, Ustekinumab or Apremilast). In the 1st period, $143(86,14 \%)$ patients were treated with TNFi and $23(13,85 \%)$ with non-TNFi; in the 2nd period, $116(78,4 \%)$ patients were treated with TNFi and $32(21,2 \%)$ with non-TNFi. PsA patients with polyarticular involvement were on TNFi in a higher prevalence in the first period than in the second $(p<0.001)$. On the contrary, oligo-articular PsA and axial PsA were on TNFi in a higher prevalence in the 2nd period than in the 1 st $(p<0.001)$, as well as non-TNFi drugs were more frequently used in the 2 nd period than in the $1 \mathrm{st}$ $(p=0.03)$. PsA patients with PsO and/or onychopathy were in a higher prevalence on non-TNFi than on TNFi in the 1st period $(p<0.001)$. PsA with enthesitis and/or dactylitis were in a higher prevalence on TNFi than on non-TNFi in the 1 st period $(p<0.001)$; this difference disappeared in the 2nd period, when both TNFi and non-TNFi were equally used ( $\mathrm{p}=\mathrm{ns}$ ). PsA patients with metabolic syndrome and/or cardio-vascular and/or renal diseases were more prevalent on TNFi in the 1st period than in the 2nd while in the 2nd these patients were more frequently on non-TNFi $(p<0.05)$.

Conclusion: Here, we demonstrated that clinicians treatment choice in patients with moderate-severe PsA may be influenced by the clinical subtype and/or the occurrence of comorbidities. In particular, during the last years, the use of nonTNFi can be chosen more adequately as a therapeutical challenge in patients with different clinical manifestations and with comorbidities, improving PsA treatment paradigm.

Table 1. Clinical characteristics of patients enrolled.

\begin{tabular}{lcccc}
\hline & \multicolumn{3}{c}{ TNFi } & \multicolumn{3}{c}{ Non-TNFi } \\
\cline { 2 - 5 } & $2004-2011$ & $2012-2019$ & $2004-2011$ & $2012-2019$ \\
\hline F (N) & 104 & 68 & 16 & 22 \\
M (N) & 53 & 34 & 9 & 8 \\
Total, divided for period & 157 & 102 & 25 & 30 \\
Total, divided for drugs & 259 & & 55 & \\
F/M (\%) & $66 / 34$ & $67 / 33$ & $64 / 36$ & $73 / 27$ \\
Age, years & $55,4 \pm 13,09$ & $56,28 \pm 11,34$ & $54,80 \pm 12,56$ & $57,44 \pm 11,15$ \\
Disease duration, months & $156 \pm 106,03$ & $162,69 \pm 101,65$ & $172,87 \pm 108,53$ & $167,2 \pm 108,89$ \\
Age of onset, years & $42,57 \pm 13,00$ & $41,73 \pm 12,13$ & $42,065 \pm 15,33$ & $45,8 \pm 13,6$ \\
Age of diagnosis, years & $44,74 \pm 12,98$ & $43,35 \pm 11,25$ & $43,4 \pm 14,33$ & $46,8 \pm 15,98$ \\
Diagnostic delay, months & $72 \pm 34,68$ & $12 \pm 8,52$ & $6,73 \pm 10,64$ & $27 \pm 15,12$ \\
\hline
\end{tabular}

Disclosure of Interests: None declared

DOI: 10.1136/annrheumdis-2020-eular.5403

\section{$\mathrm{AB} 0755$ \\ REAL WORLD EFFECTIVENESS OF SECUKINUMAB IN PSORIATIC ARTHRITIS: FINDINGS FROM A RECENT CROSS SECTIONAL SURVEY OF RHEUMATOLOGISTS AND PATIENTS IN EUROPE}

P. G. Conaghan ${ }^{1}$, E. Holdsworth ${ }^{2}$, H. Tian ${ }^{3}$, N. Booth ${ }^{2}$, P. Anthony ${ }^{2}$, N. Modi ${ }^{4}$,

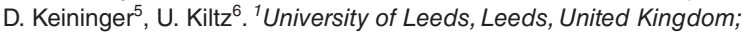

${ }^{2}$ Adelphi Real World, Bollington, United Kingdom; ${ }^{3}$ Novartis Pharmaceuticals
Corporation, East Hanover, NJ, United States of America; ${ }^{4}$ Novartis Healthcare Pvt Ltd, Hyderabad, India; ${ }^{5}$ Novartis Pharma AG, Basel, Switzerland;

${ }^{6}$ Rheumazentrum Ruhrgebiet, Herne, and Ruhr-University Bochum, Bochum, Germany

Background: Secukinumab has demonstrated significant and sustained reduction of disease activity and improvement in physical functioning and quality of life in PsA pts in RCTs. ${ }^{1}$

Objectives: This study assessed effectiveness of secukinumab in PsA in a realworld setting.

Methods: This was a cross-sectional survey of rheumatologists, dermatologists and pts in France, Germany, Italy, Spain, and UK. Data were collected online from June-Dec 2018 via physician-completed patient record forms. Pts receiving any treatment for PsA were included in survey $(n=1675)$. Pts receiving secukinumab $>4$ months were included in this analysis. Pts reported quality of life, work, and disability measures at their current consultation. Physicians reported patient demographic and disease characteristics, concomitant and previous treatments, and time since diagnosis. Physicians also reported overall, skin and joint disease severity, pain (1-10 scale), BSA psoriasis involvement, global VAS score, PAS I score, SJC, and TJC for 2 time points: initiation of treatment and at the time of data collection (current consultation). Data were analysed descriptively. The data analysed here is representative of pts that are currently receiving secukinumab and does not assess pts that have discontinued treatment.

Results: 572 PsA pts were receiving secukinumab $>4$ months at their curren consultation. Patient mean age was $47.9 \mathrm{yrs}$, with $43 \%$ female, $59 \%$ working full time, and a mean BMI of 26.6. On average, pts were diagnosed with PsA 5.6 years before the current consultation, had received secukinumab for 11.0 months, and for $59 \%$ of pts secukinumab was their 1st advanced therapy (bDMARDs or tsDMARDs), $24 \%$ their 2 nd and $16 \%$ their 3 rd or more. $25 \%$ of pts were also receiving a csDMARD concurrently. Pts reported a mean EQ5D utility score of 0.83 , mean WPAI overall work impairment of $24.3 \%$, mean HAQ-DI score as 0.6 , and mean PsAID12 score as 2.6 at current consultation. Proportion of pts with moderate and severe overall disease severity, and skin and joint severity decreased at current consultation vs at the initiation treatment (Table 1). Between initiation of treatment and current consultation, pts achieved a significant reduction in disease activity scores, pain score, global VAS scores, BSA, PASI score as well as a greater proportion of pts achieving a BSA $<3 \%$, a PASI score $<3$ (Table 2)

Conclusion: This multinational study demonstrated secukinumab effectiveness in routine care in PsA pts, with significant improvements across all outcomes. References:

[1] Mease et al. RMD Open. 2018; 4(2): e000723

Table 1. Disease severity at initiation of secukinumab and at current consultation

\begin{tabular}{lcc}
\hline & $\begin{array}{c}\text { At initiation of } \\
\text { secukinumab ( } \mathbf{n = 5 7 2 )}\end{array}$ & $\begin{array}{c}\text { At current } \\
\text { consultation ( } \mathbf{n = 5 7 2 )}\end{array}$ \\
\hline Overall disease severity, $\mathrm{n}(\%)$ & & \\
\hline Mild & $32(5.6)$ & $432(75.5)$ \\
Moderate & $316(55.2)$ & $131(22.9)$ \\
Severe & $218(38.1)$ & $9(1.6)$ \\
Don't know & $6(1.0)$ & - \\
Skin severity, $\mathrm{n}(\%)$ & & $478(83.6)$ \\
Mild & $93(16.3)$ & $82(14.3)$ \\
Moderate & $287(51.2)$ & $12(2.1)$ \\
Severe & $154(26.9)$ & - \\
Don't know & $38(6.6$ & $444(77.6)$ \\
Joint severity, $\mathrm{n}(\%)$ & & $118(20.6)$ \\
Mild & $50(8.7)$ & $10(1.7)$ \\
Moderate & $329(57.5)$ & - \\
Severe & $181(31.6)$ & \\
Don't know & $12(2.1)$ & \\
\hline
\end{tabular}

Table 2. Physician reported outcomes at initiation of secukinumab and at current consultation, mean (SD)

\begin{tabular}{lcc}
\hline & $\begin{array}{c}\text { At initiation of } \\
\text { secukinumab }(\mathbf{n = 5 7 2 )}\end{array}$ & $\begin{array}{c}\text { At current } \\
\text { consultation }(\mathbf{n = 5 7 2})\end{array}$ \\
\hline BSA $^{\mathrm{a}}$ & $19.2(15.3)$ & $4.6(8.7)$ \\
$\mathrm{BSA}<3 \%, \mathrm{n}(\%)$ & $28(8.2)$ & $213(62.5)$ \\
PASI score (0-72) & $17.2(11.5)$ & $4.2(8)$ \\
PASI score $<3, \mathrm{n}(\%)$ & $11(7.1)$ & $99(64.3)$ \\
DAS28 score & $5.2(1.5)^{\star}$ & $2.8(1.3)$ \\
TJC $(0-68)^{\mathrm{a}}$ & $12.1(9.9)$ & $2.5(3.6)$ \\
SJC $(0-66)^{\mathrm{a}}$ & $10.0(9.5)$ & $2.9(7.5)$ \\
Pain score $(1-10)$ & $6.3(2.0)$ & $2.6(1.6)$ \\
${\text { Physician global VAS score }(1-100)^{\mathrm{a}}}^{\mathrm{a}}$ & $59.4(24.2)$ & $23.3(22.2)$
\end{tabular}

